\title{
Klaus König
}

\section{Sobre la evaluación de los programas estatales $(*)$}

La cuestión de la eficacia de los programas estatales -incluida aquí, y muy particularmente, la de las leyes - está adquiriendo cada día mayor actualidad. No sólo en la República Federal de Alemania (RFA), sino también en otros muchos Estados democráticos occidentales parace haber desaparecido la confianza en la capacidad estatal de dirección y regulación. Si el fracaso del mercado fue en su día la razón que se adujo para justificar la intervención estatal, hoy el interés se centra más en el mal funcionamiento de la política, la legislación y la Administración. Refiriéndonos a la legislación, vemos que las leyes, medidas por sus propios objetivos, revelan a menudo un alto grado de inefectividad y de efectos secundarios negativos, así como una disminución de la disposición de cumplimiento por parte del ciudadano, e incluso por la propia Administración (1). Todo ello es básicamente consecuencia de la marea legislativa existente, así como del extremado perfeccionismo regulativo. Algunos autores no se limitan en este aspecto a una crítica de los programas y estrategias estatales, sino que, yendo

${ }^{\star}{ }^{\star}$ Artículo publicado en la revista Schriften zur Öffentlichen Verwaltung und Öffentlichen Wirtchaft: Erfolgskontrolle bei der Vervausgabung Öffentliches Mittel, tomo 91, pp. 19-34. El INAP y la Redacción de DOCUMENTACIÓN ADMINISTRATIVA desean dejar constancia de su agradecimiento al autor y editores, por la autorización concedida para la traducción al castellano y reproducción del presente trabajo.

(1) Cfr. Thomas FLEINER, «Norm und Wirklichkeit», en Zeitscbrift für Schweitzerisches Recbt, tomo II, 1974, pp. 279 y ss.; Erhard BLANKENBURG, «Über die Unwirksamkeit von Gesetzen», en Archiv für Rechts-und Sozialphilosopbie, 1977, pp. 44 y ss. 
más allá, hablan de una sobrecarga excesiva del Estado (2), y plantean el problema de la determinación de los límites del gobierno en una democracia parlamentaria (3).

Con lo cual se pone claramente de relieve la íntima conexión que media entre las cuestiones que afectan a la eficacia de los programas estatales, a saber: el concepto fundamental de la relación entre el Estado y la sociedad y la función de dirección que compete a un Estado moderno social y de Derecho.

Un Estado de prestaciones y redistributivo no puede limitarse simplemente a fijar, a través de las regulaciones legislativas, el marco para las actividades de la sociedad, sino que se impone la necesidad de una actuación dirigente del gobierno y la Administración. Los principios abstractos de igualdad y universalidad no pueden ser los únicos pilares en que se apoyen unas leyes que dirigen programáticamente la influencia del Estado sobre los procesos sociales, sino que habrán de regularse explícitamente con vistas a los objetivos concretos perseguidos. Así, la vieja cuestión sobre si el Derecho es un medio o un fin (en sí mismo) encuentra una respuesta con su instrumentalización. La legislación se convierte en dirección de programación política (4) y la Administración en el desarrollo de la misma. Con lo cual - como la legislación está sometida a una aceptación política determinada- las interrelaciones de los efectos serán determinantes a la hora de valorar los mismos. Y aun así, nos encontramos ante una situación un tanto paradójica, a saber: justamente lo relativo a las interconexiones de causa-efecto pasa aquí a un segundo plano en las discusiones y consultas políticas de las leyes o de los otros programas estatales de actuación. Se dice que las leyes suelen actuar de acuerdo a sus objetivos pretendidos. Y ello se deriva, en resumidas cuentas, más bien de las condiciones de nuestro sistema político de representación y no tanto de una confianza en las propias normas. Como los efectos reales de un programa estatal no suelen poder contrastarse más que a largo plazo, hasta ese momento todo lo demás queda relegado a meras conjeturas. Mientras tanto, el elector va decidiendo a un ritmo cuatrienal parlamentario sobre los éxitos y fracasos del gobierno. Con la consecuencia de que los éxitos del gobierno se decidirán a través de las leyes aprobadas, o viceversa, y se pondrán en duda por referencia a la no aprobación de determinadas leyes o al fracaso de las publicadas.

(2) Cfr. Niklas LuHMaNN, «Die Organisationsmittel des Wohlfahrtstaates und ihre Grenzen», en Heiner GeISSLER (ed.), Verwaltete Bürger - Gesellschaft in Fesseln, Berlín, 1978, pp. 112 y ss.; Helmut KLAGES, Überlasteter Staat, verdrossene Bürger?, $Z u$ den Dissonanzen der Wobl. fabrtsgesellschaft, Frankfurt am Main, 1981.

(3) Cfr. Frqanz LeHNER, Grezen des Regierens, Königstein/Taunus, 1979.

(4) Cfr. Klaus KÖNIG, «Programmsteuerung im komplexen politischen Systemen", en Die Vervaltung, 1974, pp. 137 y ss. 
De igual manera vemos cómo los programas del partido se convierten automáticamente en gubernamentales y éstos, a su vez, compondrán el programa de un período legislativo, mediante el cual se pretenderán «realizar» las declaraciones del gobierno. Y todo el círculo que va desde las elecciones, la mayoría parlamentaria, la formación de gobierno, legislación, desarrollo legislativo y las nuevas elecciones se constituye así, fundamentalmente, sobre la suposición de la efectividad de las leyes. Sin darse ésta, sería prácticamente imposible determinar la relación existente entre la decisión electoral y los efectos que se le imputan (5).

Esta supuesta imputación de la efectividad de las leyes —derivada ciertamente del sistema - puede quedar respaldada en varios supuestos por el estudio y reconocimiento de las conexiones entre causaefecto. Hasta qué punto eso se da realmente dependerá en última instancia de la politización misma de tales causas y efectos. $Y$ así, en algunos ámbitos políticos, como sería la protección del medio ambiente, la polémica en torno a los daños y perjuicios forestales se ha convertido en objeto de una discusión que excede del círculo meramente técnico o de expertos. Y como consecuencia de ello, se llevan a cabo tests muy completos sobre las posibles medidas a realizar, como, por ejemplo, sobre la limitación de la velocidad. A esto se le une una importante discusión sobre los métodos de influencia estatal, que llegó a ser un día tema de las deliberaciones del Gabinete. Por otro lado, en la legislación social suele centrarse la atención política en la incidencia social y en la igualdad, descuidando un tanto los problemas de la efectividad.

Se quiere llamar asimismo la atención sobre los límites de una concepción instrumental de las leyes que, aunque sean estrictamente finalista, siguen conservando su naturaleza de normas jurídicas y a las que no se pueden negar sus características jurídicas más esenciales. Cada instrumento jurídico habrá de ser relativizado desde la perspectiva de la Justicia (6).

Con todo lo cual se pone de manifiesto que la mera relación de causa-efecto es insuficiente como única orientación para la actividad legislativa, pues no podrá eliminarse nunca del todo la inseguridad existente sobre la eficacia de los instrumentos político-administrativos de dirección, dado que la actividad social es prácticamente indeterminable (7).

(5) Cfr. Wolfgang ZEH, Wille und Wirkung der Gesetze, Heidelberg, 1984.

(6) Cfr. Hans RyFfeL, Rechstsoziologie, Neuwied und Berlin, 1974.

(7) Cfr. Waldemar SCHRECKENBERGER, «Sozialer Wandel als Problem der Gesetzgebung», en Vewaltungsarchiv, 1977, pp. 28 y ss. 
En una búsqueda de los posibles aspectos de mejora de los programas estatales, a través de evaluaciones ex-ante, no podrán dejarse de lado los límites de una orientación de eficacia de la política.

A finales de los años sesenta y principios de los setenta se desarrolló una política que, a pesar de conocerse con el nombre de «política experimental» (8), es dudoso que tuviera suficientemente en cuenta los límites citados. Se contaba entonces con una política de reformas y con ello pasó a primer plano un concepto de política que se basaba en un doble presupuesto, a saber: por un lado, se creía en la posibilidad de una política «activa», y por otro, se partía de una alta capacidad de aprendizaje de ésta. Esto debía verse acompañado por todo tipo de experimentos sociales, pruebas modelo y otra clase de evaluaciones. Sirvan como ejemplo para estos proyectos de política experimental los ensayos de reformas escolares y demás propuestas en el ámbito educativo que en aquella época se realizaron.

Este modelo de evaluación intensificada que debía acompañar a los programas estatales recibió un fuerte impulso tras los esfuerzos de institucionalizar, tanto en el Bund como en los Läder, sistemas de planificación (9), ya que las evaluaciones se enmarcaban dentro de un ciclo global de planificación y management (10). Estos proyectos de planificación en el Bund han sido descritos sucintamente, así como el análisis en detalle de las razones de su fracaso. Debiendo ser tenidas en cuenta estas experiencias en futuras evaluaciones del proceso político (11).

En este marco de planificación política se han llevado a cabo diferentes valoraciones, análisis de coste-beneficio y coste-efectividad, que tratan de encauzar las consecuencias de diferentes decisiones en ámbitos políticos distintos, cuantificándolos para ofrecer unos datos que puedan ser comparados entre sí «objetivamente». Pero se trata aquí de un método que queda limitado por sus propias premisas y por las condiciones políticas dadas. Este tipo de procedimiento sólo es practicable si existe previamente la posibilidad de una comparación obje-

(8) Cfr. Gerd-Michael Hellstern y Hellmut WollmanN (eds.), Experimentelle Politik Reformstroghfeuer oder Lernstrategie, Opladen, 1983.

(9) Cfr. Fritz W. SCHARPF, Planung als politischer Prozess, Frankfurt am Main, 1973.

(10) Cfr. Rudolf STEINBERG, «Evaluation als neue Form der Kontrolle final programierten Verwaltungshandelns», en Der Staat, 1976, pp. 185 y ss.; Hans-Ulrich DerLIEN, Die Erfolgskontrolle staatlicher Planung, Eine empirische Untersuchung über Organisationen, Methode und Politik der Programmevaluation, Schriften zur öffentlichen Verwaltung und öffentlichen Wirtschaft, editado por Peter EichHORN y Peter Friedrich, tomo 17, Baden-Baden, 1976.

(11) Cfr. Rudolf DiECKMANN, «Schwierigkeiten mit der Erfolgskontrolle in der öffentlichen Verwaltung», en Die öffentliche Verwaltung, 1980, pp. 737 y ss. 
tivizada de las distintas consecuencias que se dan para grupos distintos. $\mathrm{Y}$ es aquí donde radica su propia imposibilidad, ya que no existe ningún procedimiento en el que pueda ser objetivizada la comparación entre tipos diferentes de decisiones (como, por ejemplo, la mejora del tráfico o el saneamiento de las rentas), o en momentos y para grupos diferentes (como subvenciones para la industria del acero o para la agricultura). A ello se suma que las técnicas de optimización han de presuponer un factor decisorio unitario que pueda ordenar sin contradicciones sus propias preferencias. La situación política ofrece, sin embargo, una pluridad de implicados que, a su vez, disponen de diferentes potenciales de poder que se rigen por sus propios objetivos e intereses. Todo ello es de tal complejidad que difícilmente puede ser recogida por las escalas valorativas que han de cuantificarlo (12).

Lo dicho no ha de conducir a la deducción errónea de que tales ponderaciones ex-ante de coste-beneficio no tengan ninguna aplicación en el ámbito estatal. Pues estudios, por ejemplo, en el sector de tráfico han demostrado que ofrecen resultados importantes para una valoración adecuada con vistas a las posibles alternativas, siempre que se trate de métodos aplicables a proyectos más bien técnicos y preparatorios. No obstante, hay que resaltar también en este lugar la función heurística de tales métodos de procedimiento de decisión política.

Para todas las técnicas de evaluación rige el principio de que un mejor uso de la información en el proceso político sólo será aprovechado en la medida en que los mismos procesos de conflicto o consenso lo exijan o, por lo menos, lo permitan. Queda constatado, por lo tanto, que las evaluaciones pueden aportar a lo sumo datos para elaborar unos procedimientos políticos más «inteligibles» y no, en cambio, para renovar sus condiciones sistemáticas.

El gobierno federal ha decidido recientemente realizar un estudio profundo sobre la necesidad, efectividad, practicabilidad y comprensión de los nuevos proyectos legislativos; lo que viene a ser una evaluación ex-ante que pretende poner de relieve la interdependencia político-administrativa (13).

Este método mencionado — decidido conjuntamente con estrategias de desburocratización y simplificación del sistema jurídico y administrativo- tiene como modelo algunos proyectos parecidos que se realizaron en los Länder. El objetivo primordial de este estudio es establecer el deber de motivar el desarrollo de los programas, tanto a nivel ministerial como interministerial, e incluso también en el ámbito de las deliberaciones del Gabinete. No se trata, pues, de la aplicación

(12) Cfr. Fritz W. SCHARPF, «Politische Planung zwischen Anspruch und Realität», en Jürgen V. JEKEwITZ (ed.), Politik als gelebte Verfassung, Opladen, 1980, pp. 93 y ss.

(13) Cfr. Bundesminister des Inneren (ed.), Erster Bericht zur Rechts-und Verwaltungsvereinfacbung, Bonn, 1985. 
de técnicas sociales para la determinación cuasicientífica de decisiones, sino que lo que se pretende es fomentar un diálogo crítico entre las distintas secciones técnicas y la sección encargada del estudio del objetivo global de simplificación del sistema jurídico y la Administración.

Un diálogo que vendrá estructurado por unas preguntas enfocadas específicamente hacia un concepto determinado por la eficacia de la legislación. De este modo se espera lograr que se estudie ya de manera más sistemática y con vistas al desarrollo de los programas:

- si realmente es preciso hacer algo,

- si existen alternativas,

- si es el Bund el que tiene que actuar,

- si hay que elaborar una ley,

- si hay que actuar en ese preciso momento,

- si las dimensiones de la regulación propuesta son necesarias,

- si la regulación está próxima al ciudadano, si es comprensible y practicable, $y$

- hasta qué punto los costes y beneficios de la regulación se encuentran en una relación acertada.

Desde la perspectiva de la efectividad merece especial atención la cuestión de las posibles alternativas a la influencia del Estado en procesos sociales (14).

En concreto aquí se pregunta (15):

- ¿Qué resultado ofrece el análisis del problema y cuáles son las causas del mismo? ¿Qué factores pueden ser cambiados?

- ¿Con qué métodos de aplicabilidad común se pueden alcanzar los objetivos de forma completa o razonablemente parcial?

- ¿Cuáles son los instrumentos de actuación más adecuados, teniendo en cuenta el esfuerzo y la carga que representan para el ciudadano y la economía, así como la eficacia, coste y gasto para los presupuestos públicos; la repercusión sobre las normas existentes, efectos secundarios; la comprensión y aceptación por parte de los destinatarios y aplicadores?

- ¿Mediante qué procedimiento se puede evitar la elaboración de nuevas normas?

La complejidad de todas estas relaciones no permite una respuesta fácil. Se confía de todas maneras en que sean aplicables las experiencias obtenidas en la evaluación de los programas estatales para obtener una mejor estimación previa sobre la efectividad de la estrategia de dirección del Estado. El análisis de la eficacia y su control preten-

(14) Cfr. Bundesminister des Inneren, op. cit., nota 13, p. 15.

(15) Cfr. Bundesminister des Inneren, op. cit., nota 13, pp. 52 y ss. 
den determinar igualmente los tipos de actividad en relación con una dirección político-administrativa, aunque queda por resolver hasta qué punto estas estrategias de actividad, elaboradas en relación a determinados ámbitos políticos, podrán ser abstraídas posteriormente. Estos temas fueron el objeto central de una reunión de expertos organizada por el Ministerio del Interior (16). Entretanto parece ser que se está alcanzando un consenso para distinguir -empleando criterios de efectividad- unos modelos de instrumentos de intervención estatales, como puedan ser incentivos, obligaciones, prohibiciones, transferencias financieras, etc. El siguiente paso para obtener más información sobre estas evaluaciones ex-ante tendría que ser la determinación (en cada caso concreto) de las diferencias de los modelos-tipo de actuación estatal y no estatal, en relación a su efectividad, sus problemas de aplicación y sus costes. Pero sobre este tema no existen más que algunas vagas ideas y habrá que profundizar en estos aspectos para obtener más datos sobre su evaluación y valoración sistemática. Sea como fuere, siempre quedará un amplio margen para las evaluaciones politicas a la hora de responder a las cuestiones fundamentales de la necesidad, efectividad y practicabilidad de los proyectos legislativos.

Estos estudios - que se elaboran con ayuda de cuestionarios y, en su caso, además, con catálogos de criterios (17) - se suelen referir en general tan sólo a aspectos parciales de los proyectos del gobierno. Para un estudio global previo del programa estatal, con vistas a su efectividad y practicabilidad, acostumbran a realizarse tests prácticos y simulaciones (18).

Los tests prácticos consisten en realizar con los futuros desarrolladores de los planes y con los afectados por los mismos una aplicación ficticia de ellos. Este tipo de test se aplicó, según lo prescrito en el Ausbildungsförderungsgesetz (Ley de Formación Profesional), por ejemplo, en el Sozialbilfegesetz (Ley de Ayuda Social), en el Jugendbilfegesetz (Ley de Ayuda a la Juventud) o en la Abgabenordnung (Ley Tributaria).

En contraposición a estos tests prácticos, en una simulación los roles de las autoridades o instancias privadas son interpretados por personas diferentes de aquéllas que más adelante los habrán de desempeñar realmente. $Y$ así, se ha aplicado en el Bundesbaugesetz (Ley Federal de Obras), el Städtebauförderungsgesetz (Ley de Fomento de la Ur-

(16) Cfr. Bundesminister des Inneren, Sachverständigenanbörung zu Ursachen einer Bürokratisierung in der öffentlichen Verwaltung sowie zu ausgewäblien Vorbaben zur Verbesserung des Verbältnisses von Bürgern und Verwaltung, Bonn, 1980; Teil A: Zusammenstellung der Schriftlinchen Stellungnahme der Sachverständigen; Teil B: Stenographisches Wortprotokoll; Teil C: Renate MAYNTZ, Wissenschaftliche Auswertung.

(17) Cfr. Harald KINDERMANN, Ministerielle Richtlinien der Gesetzestechnik, Berlin, 1979; Werner Hugger, Gesetze-inbe Vorbereitung, Abfassung und Prüfung, Baden-Baden, 1983.

(18) Cfr. Carl BÖHRET y Werner HuGGER, «Bessere Gesetze durch Test der Entwürfe?», en Zeitscbrift für Parlamentsfragen, 1979, pp. 245 y ss. 
banización Municipal) y en el Gesetz zum Abbau der Feblbelegung im sozialen Wobnungsbau (Ley para la Supresión de la Ocupación Inadecuada de las Viviendas Sociales).

Por regla general, estos tests prácticos y simulaciones de los proyectos de ley gozan de bastante aceptación, ya que gracias a ellos se descubrieron una serie de vicios y también posibilidades de perfeccionamiento que de otra manera -incluso tras un repaso o discusión más profunda - no hubiesen sido detectados (19). Algunos resultados de los tests quedaron recogidos ya directamente en una versión del proyecto. Pero sin que tampoco puedan pasarse aquí por alto los límites de los conocimientos que de esta manera se adquieren, como pueden ser, por ejemplo, los de la inevitable suposición de la efectividad real del Derecho. Además, hay que apuntar que el proceso político no quedará suspendido por la prueba de estos proyectos y que, en última instancia, los resultados estarán sometidos a valoraciones políticas.

\section{III}

Si se recurre, como ya se ha descrito, a programas estatales, y especialmente también a regulaciones legales, para alcanzar una influencia concreta sobre las condiciones sociales, esto tendrá asimismo consecuencias para los controles posteriores del programa. Pues ya no es suficiente que las leyes se cumplan, es decir, que determinados hechos queden cubiertos por la regulación legal y que se deriven de ello las consecuencias jurídicas pertinentes, sino que es necesario también el estudio de los efectos de tales leyes y la realización de evaluaciones controladoras de su resultado (20).

El estudio de su eficacia también puede llevarse a cabo en el marco de un control de su legalidad. Lo que no implica, por supuesto, que toda actividad ineficaz pueda ser calificada sin más de inválida. Otra cosa es que a los aspectos de la eficacia se les atribuyan a su vez cualidades de validez.

Así sucede, sin embargo, en el ámbito del principio de proporcionalidad, según el cual esa determinada actividad tiene que ser el medio adecuado, necesario y oportuno para la obtención del fin jurídico perseguido. Según jurisprudencia constante, este principio de proporcionalidad es un principio de carácter universal, no escrito y con rango constitucional que inspira la actividad estatal. Como consecuencia de él, el legislador deberá acordar los medios que sean adecuados y 1980.

(19) Carl BÖhret y Werner Hugger, Der Praxistest von Gesetzesentwürfen, Baden-Baden,

(20) Cfr. Klaus KöNIG, «Zur Evaluation der Gesetzesgebung», en Harald KINDERMANN (ed.), Studien zu einer Theorie der Gesetzgebung, Berlín, 1982, pp. 306 y ss. 
necesarios para alcanzar la finalidad perseguida. Por tanto habrán de ser evaluados los efectos y las regulaciones y ponderados posteriormente de acuerdo a los fines. No obstante lo dicho, el legislador se ha dejado un amplio margen de libertad en lo que a las medidas legales y a su realización programática se refiere: margen que ha de ser tenido en cuenta por los tribunales a la hora de realizar su control jurídico posterior; y como consecuencia de ello, las leyes no suelen ser anuladas por su incapacidad de alcanzar sus fines.

Un control limitado de las regulaciones respecto de su eficacia resulta hoy especialmente problemático, ya que nuestro sistema político ha alcanzado el techo de su capacidad y no puede reaccionar ante las nuevas exigencias mediante una expansión de las actividades públicas. Lo que se impondría sería, por lo tanto, una revisión de las funciones públicas para lograr nuevos márgenes de actividad para los problemas que están surgiendo en esta era de cambios. Pero cualquier gobierno que realice una crítica de sus funciones (21) se dará muy pronto cuenta de lo difícil que es la atribución de nuevas funciones. Nos encontramos ante el mismo fenómeno en el caso de agrupaciones sociales, que de por sí y en abstracto se inclinan a favor de una liberalización, pero que cambian de opinión cuando está en juego su estatuto jurídico público. Por ello se trata de una forma de evaluación política especialmente difícil. Está comprobado, empero, que en relación con la crítica de un formalismo jurídico pueden emerger políticamente algunas cuestiones de eficacia.

Entretando, el Bund y los Länder van tomando algunas iniciativas para la simplificación administrativa (22). Las decisiones del Bund se recogen en las medidas que adoptó el gobierno federal el 13 de julio de 1983 (23), según las cuales, cada Departamento deberá revisar las posibilidades de simplificación de sus disposiciones. El resultado de esta revisión, que estudia sobre todo las regulaciones en relación a sus efectos y sus problemas de aplicación, se plasmó en 140 proyectos. Así, por ejemplo, se han realizado esfuerzos para reconducir las bases urbanísticas de un nuevo Baugesetzbuch y para simplificar el Derecho económico y laboral. El objeto del Erstes Rechtsreinigungsgesetz (Primera Ley de Simplificación Jurídica), cuyo trámite parlamentario ya ha comenzado, es la eliminación de las regulaciones superfluas. En estas revisiones se puso de manifiesto que en seguida se llega a los lími-

(21) Cfr. Rudolf DIECKMANN, Aufgabenkritik in einer Gross-Siadiverwaltung unter besonderer Berücksichtigung Hamburgs, Berlín, 1977.

(22) Vid. la recopilación realizada por Jürgen VoLz, «Bürgernähe in der Verwaltung», en Verwaltungsführung, Organisation, Personalwesen, 1982, pp. 9 y ss. y 70 y ss.; Günther LEIS, «Die Bürokratisierungsdebatte: Der Stand der Auseinandersetzung», en Joachim Jens HESSE (ed.), Politikwissenchaft und Vervaltungswissenschaft, Opladen, 1982, pp. 168 y ss.

(23) Cfr. Bundesminister des Inneren, op. cit., nota 13. 
tes de estas operaciones de limpieza, alcanzando la sustancia misma y teniendo que pasar a la crítica de las funciones.

La constatación de los déficits de eficacia de los programas estatales se efectúa regularmente a través del proceso político mismo. Las instancias políticas asumen las deficiencias de eficacia como un problema social nuevo y las incluyen con proposiciones de mejora dentro de su programa de actividad. El hecho de que la gran avalancha de leyes sea, en realidad, una avalancha de cambios y que la velocidad de cambio vaya aumentando vertiginosamente (24) es un dato muy significativo sobre los déficits actuales de los programas estatales vigentes. Desde este punto de vista parece necesaria una evaluación ex-post intensiva de los programas estatales y un refuerzo de los controles de eficacia.

Pero es justamente en el ámbito de los programas estatales, y sobre todo en lo que a las leyes se refiere, donde un estudio de la eficacia tropieza con mayores problemas. Lo que parece muy claro, por ejemplo, cuando se tienen presentes las interrelaciones existentes en el ámbito que se requiere regular. Desde la perspectiva de la eficacia de una ley, lo que se pretende es convertir, mediante los efectos del programa legal, una situación de partida considerada como problema social en un objetivo digno de ser alcanzado. Pero aquí hay que tener en cuenta que el programa no actúa directamente, sino que lo hace a través de otras instancias que lo realizan, especialmente tribunales y autoridades administrativas y/o los ciudadanos en cuanto destinatarios de la norma. Esta descripción, que parece sencilla, se complica pronto si se consideran en el nivel de programa los distintos instrumentos de la dirección programática y el conjunto de las leyes y regulaciones individuales que actúan a un mismo tiempo en el ámbito de la regulación. Pensando en las instancias de realización, entrarán en juego elementos organizativos, personales y procesales, que influirán en la aplicación de la ley, creando situaciones de aplicabilidad realmente complejas, que tendrán su reflejo en la relación con el ciudadano como destinatario de las leyes. En lo que al ciudadano se refiere, también existen una serie de factores individuales y sociales que influirán en la aceptación de las normas. Es esta relación entre los distintos valores y sus efectos la que ha de ser estudiada y valorada con vistas a los objetivos legales (25).

La especificación de los objetivos de los programas estatales conlleva graves dificultades, ya que no suelen poderse deducir de unas leyes o del procedimiento legislativo unos objetivos precisos, realizables y sin contradiciones. La razón de ello suele ser el carácter político del

(24) Cfr. Edda MülleR y Werner Nuding, «Gesetzgebung - "Flut" oder "Ebbe" ", en Politische Vierteljabresscbrift, núm. 1, 1984, pp. 74 y ss.

(25) Véase sobre los factores que influyen en los efectos de la ley Wolfgang ZEH, op. cit., nota 5, pp. 481 y ss. 
desarrollo de los programas, que requiere a menudo una formulación general, vaga y ambigua. Aunque las ciencias sociales han desarrollado métodos para determinar y ordenar estos objetivos, forzoso es reconocer en estos análisis de los objetivos que las leyes no son más que una aportación a esta determinación de objetivos y, por tanto, sólo una parte de la estrategia global y compleja de su realización. Los problemas de la fijación de los objetivos legales nos remiten, a su vez, a los límites de una actividad estatal programática regida por el principio de los fines y los medios (26). La concepción de «transividad» de valores derivada de una jerarquía de objetivos es algo demasiado simple para poder abarcar con ella la complejidad de los hechos sociales.

La determinación y evaluación de los efectos estudiados no sólo son complicadas por la inseguridad que encuentran en el ámbito de los objetivos, sino también por las posibles consecuencias no deseadas que pueden provocarse. Estas no resultan siempre previsibles, pero son por lo menos tan importantes para la valoración final como una repercusión adecuada a los programas. Por regla general, no suele ser suficiente el señalamiento de los efectos materiales sino que, además, habrá que tener siempre en cuenta las condiciones temporales así como los aspectos de su distribución espacial. Para una determinación consecuente de los efectos se requieren datos con características que ofrezcan una información eficaz sobre los objetivos. Y bajo este aspecto no suelen ser suficientes las estadísticas que exigen algunas leyes. Parecen necesarias, por tanto, comprobaciones especiales, con cuya ayuda y con los indicadores necesarios se puedan hacer mensurables los efectos y el grado de realización de los objetivos (27).

Para la atribución de los cambios registrados en el ámbito de la regulación al programa hay que tener en cuenta las complejas interrelaciones entre el programa legislativo, las estructuras de implementación y los ciudadanos como destinatarios de las leyes. Junto a las características del programa $\longrightarrow$ sea, y especialmente, los instrumentos elegidos para influir en los procesos sociales, así como la elaboración de las determinaciones comunicativas- habrá que considerar particularmente los factores del comportamiento real de las instancias de aplicación y la reacción de los destinatarios y también de los afectados indirectamente. La imputación de efectos a las leyes depende, por tanto y a su vez, de la valoración que de ellas se haga. Las consecuencias insatisfactorias de las leyes pueden deberse a varias razones: la suposición errónea de relaciones de efectos, deficiencias de técnica legislativa en la elaboración de los programas o errores de las instancias de

(26) Cfr. Niklas LUHMANN, Zweckbegriff und Systemrationalität, Uber die Funktion von Zwecken in sozialen Systemen, Tübingen, 1968.

(27) Cfr, Klaus KONIG, «Evaluation als Kontrolle der Gesetzgebung», Speyerer Forschungsberichte, núm. 34, Speyer, 1983. 
aplicación, en las que a su vez puede desempeñar un papel importante la falta de practicabilidad de la ley. Resulta, por tanto, recomendable que los estudios de los efectos de las leyes se complementen con los de su realización con objeto de facilitar los problemas de imputación, fijando a su vez algunos puntos a partir de los cuales se podría mejorar la certeza del cumplimiento de la ley. Las recientes investigaciones sobre la implementación de las leyes han arrojado mucha luz sobre el concepto y alcance de la ejecución de las normas (28).

\section{IV}

En aquellos países que por su cultura jurídica y administrativa cuentan con una imporante tradición de orientación programática, nos encontramos con una práctica y una investigación de las evaluaciones especialmente desarrolladas. Así, por ejemplo, en los Estados Unidos la evaluación no es nada nuevo y de las leyes aprobadas en la etapa de 1967 a 1974 por el Congreso, cuarenta contenían normas para su evaluación. En el gobierno y la Administración americana existen unidades administrativas dedicadas especialmente a la evaluación. Pero aquí no se trata sólo de una cuestión ministerial, sino también parlamentaria. La evaluación se basa en regulaciones legales expresas, que recogen a su vez los medios económicos necesarios para su desarrollo. Como se ve, la investigación de evaluaciones se ha desarrollado intensamente en los Estados Unidos, según se refleja en las publicacions existentes. Los conocimientos adquiridos orientados hacia la aplicación complementan las experiencias prácticas de la vida jurídica y gubernamental (29).

En la República Federal de Alemania han sido las dudas sobre la oportunidad de la actuación estatal las que han inducido a que también el Parlamento prescriba varios deberes de información con objeto de poder llevar a cabo sus funciones políticas de control con mayor rigor. En 1983 existían más de ochenta supuestos en los que el gobierno federal tenía el deber de informar -impuesto por la ley o el Parlamento- en diferentes ámbitos y con una extensión y grados de cumplimiento muy variados. Estos informes son en parte globales, como pueden ser los anuales o sobre el Estado de la nación, o limitados a sectores políticos concretos, como informes económicos anuales o la-

(28) Cfr. Renate MAYNTZ (ed.), Implementation politischer Programme, Königsstein, 1980; idem, Implementation politischer Programme (II), Opladen, 1983.

(29) Sobre la valoración de las evaluaciones especialmente en los Estados Unidos, véase Carole GANZ, «Plädoyer für eine Evaluierung des Evaluierungsprozesses», en Gerd Michael HeLlSTERN y Hellmut WOLlmanN (eds.), Handbuch der Evaluierungsforscbung, tomo 1, Opladen, 1984, pp. 623 y ss. 
borales y agrícolas, pero también pueden referirse a datos que ilustren sobre el estado y la ejecución de algunas leyes y programas. Desde la perspectiva de su eficacia y de lo que significan como valoración de los resultados de las medidas legislativas, tales informes suelen ser enjuiciados muy críticamente por no pasar de ser un mero infome de actividades. Algunos de éstos han llegado a convertirse en pura rutina administrativa, cuyo mantenimiento se está poniendo en duda actualmente. A pesar de su limitación y de ser parte de la propia presentación política del gobierno, no ha de ser pasado por alto que su análisis y elaboración pueden ser muy fructíferas para la obtención de datos generalizables sobre los efectos de las medidas legislativas.

Además de estos deberes informativos recogidos en las leyes, el Parlamento ha instituido algunas variedades procedimentales para obtener las informaciones necesarias sobre la repercusión de los programas estatales. En algunos casos las interpelaciones sobre las consecuencias de algunas leyes han motivado evaluaciones por parte del gobierno federal. Las interpelaciones sobre el Derecho penal material y las aplicaciones de la pena son ejemplos significativos (30).

Estos informes y las evaluaciones motivadas por las interpelaciones parlamentarias se suelen llevar a cabo por los órganos especiales del Ministerio encargado del programa legislativo con ayuda de encuestas realizadas en las diferentes Administraciones o en algunos casos con la participación de instituciones investigadoras externas. Existen además ciertas unidades administrativas que son competentes especialmente para cuestiones de evaluación. Lo dicho es aplicable igualmente a algunos ministerios del Bund.

A este propósito nos remitimos a los estudios realizados por el $\mathrm{Mi}$ nisterio de Justicia (31). Las evaluaciones realizadas en el ámbito de la justicia suelen denominarse a menudo como investigación de los hechos jurídico o como sociología jurídica empírica. La función de las evaluaciones en este ámbito, que se llevan a cabo desde principios de los años setenta a través de dictámenes sobre la investigación de hechos jurídicos y sobre criminología, es la descripción, el análisis y, en su caso, el pronóstico de las consecuencias y efectos de las regulaciones legales. Son paradigmáticas las investigaciones que estudiaron la repercusión del Zweites Wobnraumkündigungsscbutzgesetz (Segunda Ley de Protección Contra los Desahucios de Viviendas), las consecuencias de la regulación de la protección del consumidor o los cambios jurídicos en el proceso penal (Strafverfabren): todas con resultados muy

(30) Cfr. Gerald KretschMer, «Evaluierung - von Nutzen für das Parlament?», en Gerd Michael HeLlSTERN y Hellmut WOLLMANN (eds.), op. cit., nota 29, pp. 405 y ss.

(31) Cfr. Harald v. KeMPSKI, «Bemerkungen zur Entwicklung, zum Stand und zu Schwierigkeiten bei der Evaluierung im Bundesministerium der Justiz», en Gerd Michael HeLLSTERN y Hellmut Wollmann (eds.), op. cit., nota 29, pp. 255 y ss. 
positivos. Aunque también hay que añadir aquí que la utilización de los conocimientos así adquiridos topa generalmente con dificultades derivadas de ordinario del proceso político-administrativo.

En el Ministerio Federal para la Cooperación Económica existe desde 1970 una «Sección para la Evaluación e Inspección Central» (32). Es una unidad de trabajo independiente y su misión consiste en informar a la Dirección de las constataciones realizadas y de las recomendaciones propuestas por la Inspección. Objeto de estas evaluaciones son medidas de fomento bilaterales o multilaterales en el ámbito de la política de desarrollo. Se trata aquí de proyectos y programas, pero también de modelos de cooperación, como, por ejemplo, ayuda personal a la participación de organizaciones internacionales. Es el Ministerio Federal para la Cooperación Económica el que, junto con expertos externos, lleva a cabo la evaluación, valorando tanto proyectos en curso como los ya finalizados. Los resultados se recogen en informes generales, que son comentados detenidamente con todos los participantes. La utilización real se traduce la finalización, la modificación o la conservación del proyecto o programa en cuestión así como mediante la redacción de directivas e instrucciones.

En el Ministerio para Alimentación, Agricultura y Montes se han elaborado, en relación a una nueva estructuración de orientación programática, Secciones especiales para análisis de eficacia y estudios de coste-beneficio (33). Cada Sección tiene, además, una Sección especial que se ocupa de la planificación de programas y medidas nuevas. Junto con la Sección presupuestaria, la de asuntos a largo plazo de la CE, la de análisis a largo plazo y la de análisis de eficacia quedan englobados en un grupo de planificación, que prepara las decisiones del Consejo Superior de la Sección. El objeto de estas evaluaciones son proyectos delimitados, que tienen una importancia financiera o económica muy considerable. Las investigaciones se llevan a cabo directamente por el mismo Ministerio para la Alimentación, Agricultura y Montes o por sus institutos de investigación y también por instituciones económicas o universitarias externas. Los resultados se utilizan luego como base para la modificación, suspensión o establecimiento de nuevas medidas.

A pesar de existir, como hemos visto, algunos ensayos de otro tipo de controles, la mayoría de ellos se refieren a la verificación de la legalidad y de la corrección. Y por ello existe una serie de propuestas

(32) Cfr. Rainer E. LoTZ, «Das Inspektionsreferet des Bundesministriums für wirtschaftliche Zusammenarbeit», en Gerd Michael Hellstern y Hellmut WollmanN (eds.), op. cit., nota 29 , pp. 289 y ss.

(33) Cfr. Ulrich BIELER, «Die Institutionalisierung und Handhabung von Effiziensanalysen im Bundesministerium für Ernährung, Landwirtschaft und Forsten», en Gerd Michael HeLLS. TERN y Hellmut WOLLMANN (eds.), op. cit., nota 29, pp 302 y ss. 
cuyo fin es intensificar la evaluación de programas estatales a través de medidas procesales y organizativas. Se exige, por ejemplo, una ampliación del deber de emisión de informes. $O$ se propone añadir al $\$ 7$, número 2, BHO que los estudios de coste-beneficio no se limiten a las medidas, sino que sean aplicables también a las leyes de notable importancia financiera. Se pide en relación con ello que el Tribunal $\mathrm{Fe}$ deral de Cuentas intensifique las evaluaciones de leyes con eficacia financiera (34).

Una propuesta basada en experiencias americanas quiere introducir en los programas legislativos una fecha de caducidad, para hacer depender su posterior vigencia de los fundamentos que expresamente resulten de su evaluación. Propuestas de este tipo también se han hecho ya en la República Federal de Alemania como, por ejemplo, los $\$ \$ 5 \mathrm{~b}$ y $5 \mathrm{~d}$ del Richtergesetz (Ley de Jueces), que brinda a los Länder la posibilidad de adoptar medidas durante un determinado plazo para una formación monofásica de juristas. Pero una combinación de estas evaluaciones de la limitación a un determinado plazo de validez de una ley no pasa de ser una mera excepción. Las diferencias existentes entre las distintas culturas juridicas y administrativas nos impiden un traspaso irreflexivo de los procedimientos americanos (35), cuyos requisitos de aplicación y efectos han de seguir siendo madurados. Así, por ejemplo, la comisión bávara para la reducción de tareas estatales y para la simplificación de la Administración ha denegado un Sunset-legislation, tras la evaluación de sus ventajas y desventajas.

También en la Cancillería Federal se están desarrollando proyectos para una aplicación más consecuente de las evaluaciones, en interés de una mejora de la precisión de los programas estatales. Deben ser tenidas en cuenta en este lugar las tensiones existentes entre el Departamento, el Canciller y el Gabinete. El Departamento se ocupa de la elaboración del programa, proporcionando los conocimientos materiales y la responsabilidad política. Los estudios sobre los efectos y la aplicabilidad a los programas de actuación son los mismos que los respectivos a la elaboración de programas. Una evaluación central de los programas de actividades estatales que desplieguen sus efectos externamente ha de resultar problemática, tanto por causa de su ámbito material como por su imputación política. No obstante se podría pensar en actividades tales como:

(34) Cfr! Heinz DORN, «Erfolgskontrolle durch den Bundesrechnungschof», en Gerd Michael HELLSTERN y Hellmut WOLLMANN (eds.), op. cit., nota 29, pp. 463 y ss.

(35) Cfr. Stephen J. FitzsimmoNs, «Erfahrungen aus dem Transfer von Evaluierungsforschung», en Gerd Michael HellSTERN y Hellmut WOLLMANN (eds.), op. cit., nota 29, pp. 596 y ss. 
- Recopilación en un programa de trabajo de las valoraciones realizadas por los departamentos, que quedaría luego a disposición de todos ellos.

- Recogida sistemática del estado y métodos de las evaluaciones de cada Departamento.

- Fórmulas de intercambio entre los distintos Departamentos de las evaluaciones realizadas.

De acuerdo con ello, el grupo de planificación de la Cancillería Federal ha elaborado una sinopsis sobre el estado actual de las evaluaciones de los Departamentos. Además, se ha creado un grupo de trabajo, compuesto por distintos representantes de los Ministerios, para recoger mediante procesamiento de datos los estudios sobre aplicación y efectos. Esta función coordinadora no debe excluir, por otro lado, que, de acuerdo con el «principio organizativo del Canciller», se fomente una considerable apreciación de los aspectos generales que engloban a todos los Departamentos.

Algún que otro observador de la vida pública en la República Federal de Alemania se tiene que preguntar hasta qué punto el principio de causalidad es tenido en cuenta en los debates políticos. En algunos sectores políticos se atribuye más importancia a las intuiciones que a las experiencias. $Y$ no son pocos los casos en los que las reacciones políticas son consecuencia de simples afirmaciones sobre causas y efectos; lo cual no es solucionable mediante una idea instrumental y tecnocrática de la política. Por lo demás, en nuestra cultura jurídica y administrativa es preciso enriquecer los controles existentes con variantes más orientadas hacia la eficacia. Lo que a su vez sólo será posible a través de una metodología adecuada al proceso político.

Traducción: Julia NIETo KoENIG 